\title{
On the Theory of Multilinear Singular Operators with Rough Kernels on the Weighted Morrey Spaces
}

\author{
Sha He and Xiangxing Tao \\ Department of Mathematics, Zhejiang University of Science and Technology, Hangzhou 310023, China \\ Correspondence should be addressed to Xiangxing Tao; xxtao@zust.edu.cn \\ Received 27 February 2016; Accepted 10 July 2016 \\ Academic Editor: Shijun Zheng
}

Copyright ( 92016 S. He and X. Tao. This is an open access article distributed under the Creative Commons Attribution License, which permits unrestricted use, distribution, and reproduction in any medium, provided the original work is properly cited.

We study some multilinear operators with rough kernels. For the multilinear fractional integral operators $T_{\Omega, \alpha}^{A}$ and the multilinear fractional maximal integral operators $M_{\Omega, \alpha}^{A}$, we obtain their boundedness on weighted Morrey spaces with two weights $L^{p, \kappa}(u, v)$ when $D^{\gamma} A \in \dot{\Lambda}_{\beta}(|\gamma|=m-1)$ or $D^{\gamma} A \in \mathrm{BMO}(|\gamma|=m-1)$. For the multilinear singular integral operators $T_{\Omega}^{A}$ and the multilinear maximal singular integral operators $M_{\Omega}^{A}$, we show they are bounded on weighted Morrey spaces with two weights $L^{p, \kappa}(u, v)$ if $D^{\gamma} A \in \dot{\Lambda}_{\beta}(|\gamma|=m-1)$ and bounded on weighted Morrey spaces with one weight $L^{p, \kappa}(w)$ if $D^{\gamma} A \in \operatorname{BMO}(|\gamma|=m-1)$ for $m=1,2$.

\section{Introduction and Main Results}

Let us consider the following multilinear fractional integral operator,

$$
T_{\Omega, \alpha}^{A} f(x)=\int_{\mathbb{R}^{n}} \frac{\Omega(x-y)}{|x-y|^{n-\alpha+m-1}} R_{m}(A ; x, y) f(y) d y,
$$

$$
0<\alpha<n
$$

and the multilinear fractional maximal operator:

$$
\begin{aligned}
& M_{\Omega, \alpha}^{A} f(x)=\sup _{r>0} \frac{1}{r^{n-\alpha+m-1}} \\
& \cdot \int_{|x-y|<r}\left|\Omega(x-y) R_{m}(A ; x, y) f(y)\right| d y,
\end{aligned}
$$

$$
0<\alpha<n
$$

where $\Omega \in L^{s}\left(S^{n-1}\right)(s>1)$ is homogeneous of degree zero in $\mathbb{R}^{n}, A$ is a function defined on $\mathbb{R}^{n}$, and $R_{m}(A ; x, y)$ denotes the $m$ th order Taylor series remainder of $A$ at $x$ expanded about $y$; that is,

$$
R_{m}(A ; x, y)=A(x)-\sum_{|\gamma|<m} \frac{1}{\gamma !} D^{\gamma} A(y)(x-y)^{\gamma},
$$

$\gamma=\left(\gamma_{1}, \ldots, \gamma_{n}\right)$, each $\gamma_{i}, i=1, \ldots, n$, is a nonnegative integer, $|\gamma|=\sum_{i=1}^{n} \gamma_{i}, \gamma !=\gamma_{1} ! \cdots \gamma_{n} !, x^{\gamma}=x_{1}^{\gamma_{1}} \cdots x_{n}^{\gamma_{n}}$, and $D^{\gamma}=\partial^{|\gamma|} /$ $\partial^{\gamma_{1}} x_{1} \cdots \partial^{\gamma_{n}} x_{n}$.

We notice that if $\alpha=0$, the above two operators $T_{\Omega, \alpha}^{A}$, $M_{\Omega, \alpha}^{A}$ are the multilinear singular integral operator $T_{\Omega}^{A}$ and the multilinear maximal singular integral operator $M_{\Omega}^{A}$ whose definitions are given as follows, respectively:

$$
\begin{aligned}
& T_{\Omega}^{A} f(x)=\int_{\mathbb{R}^{n}} \frac{\Omega(x-y)}{|x-y|^{n+m-1}} R_{m}(A ; x, y) f(y) d y, \\
& M_{\Omega}^{A} f(x)=\sup _{r>0} \frac{1}{r^{n+m-1}} \\
& \quad \cdot \int_{|x-y|<r}\left|\Omega(x-y) R_{m}(A ; x, y) f(y)\right| d y .
\end{aligned}
$$

For $m=1, T_{\Omega, \alpha}^{A}$ is obviously the commutator $\left[A, T_{\Omega, \alpha}\right]$ of $T_{\Omega, \alpha}$ and $A$ : $\left[A, T_{\Omega, \alpha}\right] f(x)=A(x) T_{\Omega, \alpha} f(x)-T_{\Omega, \alpha}(A f)(x)$, where $T_{\Omega, \alpha}$ is the fractional integral operator given by

$$
T_{\Omega, \alpha} f(x)=\int_{\mathbb{R}^{n}} \frac{\Omega(x-y)}{|x-y|^{n-\alpha}} f(y) d y, \quad 0<\alpha<n .
$$

There are numerous works on the study of multilinear operators with rough kernels. If $D^{\gamma} A \in \mathrm{BMO}(|\gamma|=m-1)$, 
the $L^{p}$ boundedness of $T_{\Omega}^{A}$ was obtained by means of a good- $\lambda$ inequality by Cohen and Gosselin [1]. In 1994, Hofmann [2] proved that $T_{\Omega}^{A}$ is a bounded operator on $L^{p}(w)$ when $\nabla A \epsilon$ $B M O$ and $w \in A_{p}$. Recently, Lu et al. [3] proved $T_{\Omega}^{A}$ and $M_{\Omega}^{A}$ are bounded from $L^{p}$ to $L^{q}(1 / p-1 / q=\beta / n)$ when $D^{\gamma} A \in$ $\dot{\Lambda}_{\beta}(|\gamma|=m-1)$, while for multilinear fractional integral operators, Ding and Lu [4] showed the $\left(L^{p}\left(w^{p}\right), L^{q}\left(w^{q}\right)\right)$ boundedness of $T_{\Omega, \alpha}^{A_{1}, \ldots, A_{k}}$ and $M_{\Omega, \alpha}^{A_{1}, \ldots, A_{k}}$ (their definitions will be given later) if $D^{\gamma} A_{j} \in \mathrm{BMO}(|\gamma|=m-1), j=1, \ldots, k$. After that, $\mathrm{Lu}$ and Zhang [5] proved $T_{\Omega, \alpha}^{A}$ is a bounded operator from $L^{p}$ to $L^{q}(1 / p-1 / q=(\alpha+\beta) / n)$ when $D^{\gamma} A \in$ $\dot{\Lambda}_{\beta}(|\gamma|=m-1)$.

On the other hand, the classical Morrey spaces were first introduced by Morrey [6] to study the local behavior of solutions to second-order elliptic partial differential equations. From then on, a lot of works concerning Morrey spaces and some related spaces have been done; see [7-9] and the references therein for details. In 2009, Komori and Shirai [10] first studied the weighted Morrey spaces and investigated some classical singular integrals in harmonic analysis on them, such as the Hardy-Littlewood maximal operator, the Calderón-Zygmund operator, the fractional integral operator, and the fractional maximal operator. Recently, Wang [11] discussed the boundedness of the classical singular operators with rough kernels on the weighted Morrey spaces.

We note that many works concerning $T_{\Omega, \alpha}^{A}, M_{\Omega, \alpha}^{A}, T_{\Omega}^{A}$, and $M_{\Omega}^{A}$ have been done on $L^{p}$ spaces or weighted $L^{p}$ spaces when $D^{\gamma} A$ belongs to some function spaces for $|\gamma|=m-$ 1. However, there is not any study about these operators on weighted Morrey spaces. Therefore, it is natural to ask whether they are bounded on weighted Morrey spaces. The aim of this paper is to investigate the boundedness of $T_{\Omega, \alpha}^{A}$, $M_{\Omega, \alpha}^{A}, T_{\Omega}^{A}$, and $M_{\Omega}^{A}$ on weighted Morrey spaces if $D^{\gamma} A \in$ $\dot{\Lambda}_{\beta}(|\gamma|=m-1)$ or $D^{\gamma} A \in \operatorname{BMO}(|\gamma|=m-1)$. When $D^{\gamma} A \in \dot{\Lambda}_{\beta}(|\gamma|=m-1)$, we show $T_{\Omega, \alpha}^{A}$ and $T_{\Omega}^{A}$ are controlled pointwisely by the fractional singular integral operators $\bar{T}_{\Omega, \alpha+\beta}$ and $\bar{T}_{\Omega, \beta}$ (their definition will be given later), respectively. Thus, the problem of studying the boundedness of $T_{\Omega, \alpha}^{A}$ and $T_{\Omega}^{A}$ on weighted Morrey spaces with two weights could be reduced to that of $\bar{T}_{\Omega, \alpha+\beta}$ and $\bar{T}_{\Omega, \beta}$. When $D^{\gamma} A \in \operatorname{BMO}(|\gamma|=m-1)$, the boundedness of $T_{\Omega, \alpha}^{A}$ on weighted Morrey spaces with two weights is proved by standard method. However, we could only obtain the boundedness of $T_{\Omega}^{A}$ on weighted Morrey spaces with one weight for $m=1$ and $m=2$, since we need the $L^{p}(w)$ boundedness of $T_{\Omega}^{A}$ in our proof, but to the best of our knowledge, there is not such bounds hold for $T_{\Omega}^{A}$ when $m \geq 3$. For $M_{\Omega, \alpha}^{A}$ and $M_{\Omega}^{A}$, we show they are controlled pointwisely by $T_{\Omega, \alpha}^{A}$ and $T_{\Omega}^{A}$, respectively. Thus, it is easy to obtain the same results for $M_{\Omega, \alpha}^{A}$ and $M_{\Omega}^{A}$ as those of $T_{\Omega, \alpha}^{A}$ and $T_{\Omega}^{A}$.

Before stating our main results, we introduce some definitions and notations at first.

A weight is a locally integrable function on $\mathbb{R}^{n}$ which takes values in $(0, \infty)$ almost everywhere. For a weight $w$ and a measurable set $E$, we define $w(E)=\int_{E} w(x) d x$, the Lebesgue measure of $E$ by $|E|$ and the characteristic function of $E$ by $\chi_{E}$. The weighted Lebesgue spaces with respect to the measure $w(x) d x$ are denoted by $L^{p}(w)$ with $0<p<\infty$. We say a weight $w$ satisfies the doubling condition if there exists a constant $D>0$ such that for any ball $B$, we have $w(2 B) \leq D w(B)$. When $w$ satisfies this condition, we denote $w \in \Delta_{2}$ for short.

Throughout this paper, $B\left(x_{0}, r\right)$ denotes a ball centered at $x_{0}$ with radius $r$. Let $Q$ be a cube with sides parallel to the axes. For $K>0, K Q$ denotes the cube with the same center as $Q$ and side-length being $K$ times longer. When $\alpha=0$, we will denote $T_{\Omega, \alpha}, T_{\Omega, \alpha}^{A}, M_{\Omega, \alpha}^{A}$ by $T_{\Omega}, T_{\Omega}^{A}, M_{\Omega}^{A}$, respectively. And for any number $a, a^{\prime}$ stands for the conjugate of $a$. The letter $C$ denotes a positive constant that may vary at each occurrence but is independent of the essential variable.

Next, we give the definition of weighted Morrey space introduced in [10].

Definition 1. Let $1 \leq p<\infty$, let $0<\kappa<1$, and let $w$ be a weight. Then the weighted Morrey space is defined by

$$
L^{p, \kappa}(w):=\left\{f \in L_{\mathrm{loc}}^{p}(w):\|f\|_{L^{p, \kappa}(w)}<\infty\right\},
$$

where

$$
\|f\|_{L^{p, \kappa}(w)}=\sup _{B}\left(\frac{1}{w(B)^{\kappa}} \int_{B}|f(x)|^{p} w(x) d x\right)^{1 / p},
$$

and the supremum is taken over all balls $B$ in $\mathbb{R}^{n}$.

When we investigate the boundedness of the multilinear fractional integral operator, we need to consider the weighted Morrey space with two weights. It is defined as follows.

Definition 2. Let $1 \leq p<\infty$, let $0<\kappa<1$, and let $u$, $v$ be two weights. The two weights weighted Morrey space is defined by

$$
L^{p, \kappa}(u, v):=\left\{f:\|f\|_{L^{p, \kappa}(u, v)}<\infty\right\}
$$

where

$$
\|f\|_{L^{p, \kappa}(u, v)}=\sup _{B}\left(\frac{1}{v(B)^{\kappa}} \int_{B}|f(x)|^{p} u(x) d x\right)^{1 / p},
$$

and the supremum is taken over all balls $B$ in $\mathbb{R}^{n}$. If $u=v$, then we denote $L^{p, \kappa}(u)$ for short.

As is pointed out in [10], we could also define the weighted Morrey spaces with cubes instead of balls. So we shall use these two definitions of weighted Morrey spaces appropriate to calculation.

Then, we give the definitions of Lipschitz space and $B M O$ space.

Definition 3. The Lipschitz space of $\operatorname{order} \beta, 0<\beta<1$, is defined by

$$
\dot{\Lambda}_{\beta}\left(\mathbb{R}^{n}\right)=\left\{f:|f(x)-f(y)| \leq C|x-y|^{\beta}\right\},
$$

and the smallest constant $C>0$ is the Lipschitz norm $\|\cdot\|_{\dot{\Lambda}_{\beta}}$. 
Definition 4. A locally integrable function $b$ is said to be in $\operatorname{BMO}\left(\mathbb{R}^{n}\right)$ if

$$
\|b\|_{*}=\|b\|_{\mathrm{BMO}}=\sup _{B} \frac{1}{|B|} \int_{B}\left|b(x)-b_{B}\right| d x<\infty,
$$

where

$$
b_{B}=\frac{1}{|B|} \int_{B} b(y) d y,
$$

and the supremum is taken over all balls $B$ in $\mathbb{R}^{n}$.

At last, we give the definition of two weight classes.

Definition 5. A weight function $w$ is in the Muckenhoupt class $A_{p}$ with $1<p<\infty$ if there exists $C>1$ such that for any ball $B$,

$$
\left(\frac{1}{|B|} \int_{B} w(x) d x\right)\left(\frac{1}{|B|} \int_{B} w(x)^{-1 /(p-1)} d x\right)^{p-1} \leq C .
$$

We define $A_{\infty}=\bigcup_{1<p<\infty} A_{p}$.

When $p=1$, we define $w \in A_{1}$ if there exists $C>1$ such that for almost every $x$,

$$
M w(x) \leq C w(x) .
$$

Definition 6. A weight function $w$ belongs to $A(p, q)$ for $1<$ $p<q<\infty$ if there exists $C>1$ such that such that for any ball $B$,

$$
\begin{aligned}
& \left(\frac{1}{|B|} \int_{B} w(x)^{q} d x\right)^{1 / q} \\
& \cdot\left(\frac{1}{|B|} \int_{B} w(x)^{-p /(p-1)} d x\right)^{(p-1) / p} \leq C .
\end{aligned}
$$

When $p=1$, then we define $w \in A(1, q)$ with $1<q<\infty$ if there exists $C>1$ such that

$$
\left(\frac{1}{|B|} \int_{B} w(x)^{q} d x\right)^{1 / q}\left(\text { ess } \sup _{x \in B} \frac{1}{w(x)}\right) \leq C .
$$

Remark 7 (see $[10])$. If $w \in A(p, q)$ with $1<p<q$, then

$$
\begin{aligned}
& \text { (a) } w^{q}, w^{-p^{\prime}}, w^{-q^{\prime}} \in \Delta_{2} . \\
& \text { (b) } w^{-p^{\prime}} \in A_{t^{\prime}} \text { with } t=1+q / p^{\prime} .
\end{aligned}
$$

Now we state the main results of this paper.

Theorem 8. If $0<\alpha+\beta<n, \Omega \in L^{s}\left(S^{n-1}\right)(s>1)$ is homogeneous of degree zero, $1<s^{\prime}<p<n /(\alpha+\beta)$, $1 / q=1 / p-(\alpha+\beta) / n, 0<\kappa<p / q, w^{s^{\prime}} \in A\left(p / s^{\prime}, q / s^{\prime}\right)$, $D^{\gamma} A \in \dot{\Lambda}_{\beta}(|\gamma|=m-1)$, then

$$
\begin{gathered}
\left\|T_{\Omega, \alpha}^{A} f\right\|_{L^{q, \kappa q / p}\left(w^{q}\right)} \leq C \sum_{|\gamma|=m-1}\left\|D^{\gamma} A\right\|_{\dot{\Lambda}_{\beta}}\|f\|_{L^{p, \kappa}\left(w^{p}, w^{q}\right)}, \\
\left\|M_{\Omega, \alpha}^{A} f\right\|_{L^{q, \kappa q / p}\left(w^{q}\right)} \leq C \sum_{|\gamma|=m-1}\left\|D^{\gamma} A\right\|_{\dot{\Lambda}_{\beta}}\|f\|_{L^{p, \kappa}\left(w^{p}, w^{q}\right)} .
\end{gathered}
$$

Theorem 9. If $0<\beta<1, \Omega \in L^{s}\left(S^{n-1}\right)(s>1)$ is homogeneous of degree zero, $1<s^{\prime}<p<n / \beta, 1 / q=$ $1 / p-\beta / n, 0<\kappa<p / q, w^{s^{\prime}} \in A\left(p / s^{\prime}, q / s^{\prime}\right), D^{\gamma} A \in \dot{\Lambda}_{\beta}(|\gamma|=$ $m-1)$, then

$$
\begin{gathered}
\left\|T_{\Omega}^{A} f\right\|_{L^{q, \kappa q / p}\left(w^{q}\right)} \leq C \sum_{|\gamma|=m-1}\left\|D^{\gamma} A\right\|_{\dot{\Lambda}_{\beta}}\|f\|_{L^{p, \kappa}\left(w^{p}, w^{q}\right)}, \\
\left\|M_{\Omega}^{A} f\right\|_{L^{q, \kappa q / p}\left(w^{q}\right)} \leq C \sum_{|\gamma|=m-1}\left\|D^{\gamma} A\right\|_{\dot{\Lambda}_{\beta}}\|f\|_{L^{p, \kappa}\left(w^{p}, w^{q}\right)} .
\end{gathered}
$$

Theorem 10. If $0<\alpha<n, \Omega \in L^{s}\left(S^{n-1}\right)(s>1)$ is homogeneous of degree zero, $1<s^{\prime}<p<n / \alpha, 1 / q=1 / p-\alpha / n$, $0<\kappa<p / q, w^{s^{\prime}} \in A\left(p / s^{\prime}, q / s^{\prime}\right), D^{\gamma} A \in \operatorname{BMO}(|\gamma|=m-1)$, then

$$
\begin{gathered}
\left\|T_{\Omega, \alpha}^{A} f\right\|_{L^{q, \kappa q / p}\left(w^{q}\right)} \leq C \sum_{|\gamma|=m-1}\left\|D^{\gamma} A\right\|_{*}\|f\|_{L^{p, \kappa}\left(w^{p}, w^{q}\right)}, \\
\left\|M_{\Omega, \alpha}^{A} f\right\|_{L^{q, \kappa q / p}\left(w^{q}\right)} \leq C \sum_{|\gamma|=m-1}\left\|D^{\gamma} A\right\|_{*}\|f\|_{L^{p, \kappa}\left(w^{p}, w^{q}\right)} .
\end{gathered}
$$

When $m=1$ and $m=2$, we denote $T_{\Omega}^{A}, M_{\Omega}^{A}$ by $\left[A, T_{\Omega}\right]$, $\left[A, M_{\Omega}\right]$, and $\widetilde{T}_{\Omega}^{A}, \widetilde{M}_{\Omega}^{A}$, respectively, in order to distinguish from $T_{\Omega}^{A}$ and $M_{\Omega}^{A}$ that are defined for any $m \in \mathbb{N}^{*}$. To be more precise,

$$
\begin{aligned}
& {\left[A, T_{\Omega}\right] f(x)=\int_{\mathbb{R}^{n}} \frac{\Omega(x-y)}{|x-y|^{n}}(A(x)-A(y))} \\
& \cdot f(y) d y, \\
& {\left[A, M_{\Omega}\right] f(x)=\sup _{r>0} \frac{1}{r^{n}} \int_{|x-y|<r} \Omega(x-y)} \\
& \cdot(A(x)-A(y)) f(y) d y, \\
& \widetilde{T}_{\Omega}^{A} f(x)=\int_{\mathbb{R}^{n}} \frac{\Omega(x-y)}{|x-y|^{n+1}}(A(x)-A(y)-\nabla A(y) \\
& \cdot(x-y)) f(y) d y, \\
& \widetilde{M}_{\Omega}^{A} f(x)=\sup _{r>0} \frac{1}{r^{n+1}} \int_{|x-y|<r} \Omega(x-y) \\
& \cdot(A(x)-A(y)-\nabla A(y)(x-y)) f(y) d y .
\end{aligned}
$$

Then for the above operators, we have the following results on weighted Morrey spaces with one weight.

Theorem 11. If $\Omega \in L^{s}\left(S^{n-1}\right)(s>1)$ is homogeneous of degree zero and satisfies the vanishing condition $\int_{S^{n-1}} \Omega\left(x^{\prime}\right) d \sigma\left(x^{\prime}\right)=$ $0,1<s^{\prime}<p<\infty, 0<\kappa<1, w \in A_{p / s^{\prime}}, A \in \mathrm{BMO}$, then

$$
\begin{aligned}
\left\|\left[A, T_{\Omega}\right] f\right\|_{L^{p, \kappa}(w)} & \leq C\|A\|_{*}\|f\|_{L^{p, \kappa}(w)}, \\
\left\|\left[A, M_{\Omega}\right] f\right\|_{L^{p, \kappa}(w)} & \leq C\|A\|_{*}\|f\|_{L^{p, \kappa}(w)} .
\end{aligned}
$$


Theorem 12. If $\Omega \in L^{\infty}\left(S^{n-1}\right)$ is homogeneous of degree zero and satisfies the moment condition $\int_{S^{n-1}} \theta \Omega(\theta) d \theta=0,1<p<$ $\infty, 0<\kappa<1, w \in A_{p}, \nabla A \in \mathrm{BMO}$, then

$$
\begin{aligned}
\left\|\widetilde{T}_{\Omega}^{A} f\right\|_{L^{p, \kappa}(w)} & \leq C\|\nabla A\|_{*}\|f\|_{L^{p, \kappa}(w)}, \\
\left\|\widetilde{M}_{\Omega}^{A} f\right\|_{L^{p, \kappa}(w)} & \leq C\|\nabla A\|_{*}\|f\|_{L^{p, \kappa}(w)} .
\end{aligned}
$$

Remark 13. Here we point out that for $T_{\Omega}^{A}$ and $M_{\Omega}^{A}$, when $D^{\gamma} A \in \mathrm{BMO}(|\gamma|=m-1)$, the analogues of Theorems 11 and 12 are open for $m \geq 3$.

Remark 14. Define

$$
\begin{aligned}
& T_{\Omega, \alpha}^{A_{1}, \ldots, A_{k}} f(x)=\int_{\mathbb{R}^{n}} \prod_{i=1}^{k} R_{m_{i}}\left(A_{i} ; x, y\right) \\
& \cdot \frac{\Omega(x-y)}{|x-y|^{n-\alpha+N}} f(y) d y, \\
& M_{\Omega, \alpha}^{A_{1}, \ldots, A_{k}} f(x)=\sup _{r>0} \frac{1}{r^{n-\alpha+N}} \int_{|x-y|<r}|\Omega(x-y)| \\
& \cdot \prod_{i=1}^{k}\left|R_{m_{i}}\left(A_{i} ; x, y\right)\right||f(y)| d y,
\end{aligned}
$$

where $R_{m_{i}}\left(A_{i} ; x, y\right)=A_{i}(x)-\sum_{|\gamma|<m_{i}}(1 / \gamma !) D^{\gamma} A_{i}(y)(x-$ $y)^{\gamma}, i=1, \ldots, k, N=\sum_{i=1}^{k}\left(m_{i}-1\right)$. When $0<\alpha<n$, they are a class of multilinear fractional integral operators and multilinear fractional maximal operators. When $\alpha=0$, they are a class of multilinear singular integral operators and multilinear maximal singular integral operators. Repeating the proofs of the theorems above, we will find that for $T_{\Omega, \alpha}^{A_{1}, \ldots, A_{k}}$ and $M_{\Omega, \alpha}^{A_{1}, \ldots, A_{k}}$, the conclusions of Theorems 8 and 9 above with the bounds $C \prod_{i=1}^{k}\left(\sum_{|\gamma|=m_{i}-1}\left\|D^{\gamma} A_{i}\right\|_{\dot{\Lambda}_{\beta}}\right)$ and Theorem 10 with the bounds $C \prod_{i=1}^{k}\left(\sum_{|\gamma|=m_{i}-1}\left\|D^{\gamma} A_{i}\right\|_{*}\right)$ also hold, respectively.

The organization of this paper is as follows. In Section 2, we give some requisite lemmas and well-known results that are important in proving the theorems. The proof of the theorems will be shown in Section 3.

\section{Lemmas and Well-Known Results}

Lemma 15 (see [1]). Let $A(x)$ be a function on $\mathbb{R}^{n}$ with mth order derivatives in $L_{\mathrm{loc}}^{l}\left(\mathbb{R}^{n}\right)$ for somel $>n$. Then

$$
\begin{aligned}
& \left|R_{m}(A ; x, y)\right| \\
& \quad \leq C|x-y|^{m} \sum_{|\gamma|=m}\left(\frac{1}{\left|I_{x}^{y}\right|} \int_{I_{x}^{y}}\left|D^{\gamma} A(z)\right|^{l} d z\right)^{1 / l},
\end{aligned}
$$

where $I_{x}^{y}$ is the cube centered at $x$ with sides parallel to the axes, whose diameter is $5 \sqrt{n}|x-y|$.
Lemma 16 (see [12]). For $0<\beta<1,1 \leq q<\infty$, we have

$$
\begin{aligned}
\|f\|_{\dot{\Lambda}_{\beta}} & \approx \sup _{\mathrm{Q}} \frac{1}{|Q|^{1+\beta / n}} \int_{Q}\left|f(x)-b_{\mathrm{Q}}(f)\right| d x \\
& \approx \sup _{\mathrm{Q}} \frac{1}{|\mathrm{Q}|^{\beta / n}}\left(\frac{1}{|\mathrm{Q}|} \int_{\mathrm{Q}}\left|f(x)-b_{\mathrm{Q}}(f)\right|^{q} d x\right)^{1 / q} .
\end{aligned}
$$

For $q=\infty$, the formula should be interpreted appropriately.

Lemma 17 (see [13]). Let $Q_{1} \subset Q_{2}, g \in \dot{\Lambda}_{\beta}(0<\beta<1)$. Then

$$
\left|g_{\mathrm{Q}_{1}}-g_{\mathrm{Q}_{2}}\right| \leq C\left|Q_{2}\right|^{\beta / n}\|g\|_{\dot{\Lambda}_{\beta}} .
$$

Theorem 18 (see [14]). Suppose that $0<\alpha<n, 1<p<n / \alpha$, $1 / q=1 / p-\alpha / n$, and $\Omega \in L^{s}\left(S^{n-1}\right)(s>1)$ is homogeneous of degree zero. Then $T_{\Omega, \alpha}$ is a bounded operator from $L^{p}\left(w^{p}\right)$ to $L^{q}\left(w^{q}\right)$, if the index set $\{\alpha, p, q, s\}$ satisfies one of the following conditions:
(a) $s^{\prime}<p$ and $w(x)^{s^{\prime}} \in A\left(p / s^{\prime}, q / s^{\prime}\right)$;
(b) $s>q$ and $w(x)^{-s^{\prime}} \in A\left(q^{\prime} / s^{\prime}, p^{\prime} / s^{\prime}\right)$;
(c) $\alpha / n+1 / s<1 / p<1 / s^{\prime}$ and there is $r, 1<r<s /(n / \alpha)^{\prime}$ such that $w(x)^{r^{\prime}} \in A(p, q)$.

Lemma 19 (see [10]). If $w \in \Delta_{2}$, then there exists a constant $D_{1}>1$, such that

$$
w(2 B) \geq D_{1} w(B) .
$$

We call $D_{1}$ the reverse doubling constant.

Theorem 20 (see [4]). Suppose that $0<\alpha<n, 1<p<n / \alpha$, $1 / q=1 / p-\alpha / n, \Omega \in L^{s}\left(S^{n-1}\right)(s>1)$ is homogeneous of degree zero. Moreover, for $1 \leq i \leq k,|\gamma|=m_{i}-1, m_{i} \geq 2$, and $D^{\gamma} A_{i} \in \mathrm{BMO}\left(\mathbb{R}^{n}\right)$, if the index set $\{\alpha, p, q, s\}$ satisfies one of the following conditions:
(a) $s^{\prime}<p$ and $w(x)^{s^{\prime}} \in A\left(p / s^{\prime}, q / s^{\prime}\right)$;
(b) $s>q$ and $w(x)^{-s^{\prime}} \in A\left(q^{\prime} / s^{\prime}, p^{\prime} / s^{\prime}\right)$;
(c) $\alpha / n+1 / s<1 / p<1 / s^{\prime}$ and there is $r, 1<r<s /(n / \alpha)^{\prime}$, such that $w(x)^{r^{\prime}} \in A(p, q)$.

Then there is a $C>0$, independent of $f$ and $A_{i}$, such that

$$
\begin{gathered}
\left(\int_{\mathbb{R}^{n}}\left|T_{\Omega, \alpha}^{A_{1}, \ldots, A_{k}} f(x) w(x)\right|^{q} d x\right)^{1 / q} \\
\leq C \prod_{i=1}^{k}\left(\sum_{|\gamma|=m_{i}-1}\left\|D^{\gamma} A_{i}\right\|_{*}\right) \\
\cdot\left(\int_{\mathbb{R}^{n}}|f(x) w(x)|^{p} d x\right)^{1 / p} .
\end{gathered}
$$

Lemma 21 (see [15]). (a) (John-Nirenberg Lemma) Let $1 \leq$ $p<\infty$. Then $b \in \mathrm{BMO}$ if and only if

$$
\frac{1}{|Q|} \int_{Q}\left|b-b_{\mathrm{Q}}\right|^{p} d x \leq C\|b\|_{*}^{p} .
$$


(b) Assume $b \in \mathrm{BMO}$; then for cubes $\mathrm{Q}_{1} \subset \mathrm{Q}_{2}$,

$$
\left|b_{\mathrm{Q}_{1}}-b_{\mathrm{Q}_{2}}\right| \leq C \log \left(\frac{\left|\mathrm{Q}_{2}\right|}{\left|\mathrm{Q}_{1}\right|}\right)\|b\|_{*} .
$$

(c) If $b \in \mathrm{BMO}$, then

$$
\left|b_{2^{j+1} B}-b_{B}\right| \leq 2^{n}(j+1)\|b\|_{*} .
$$

Theorem 22 (see [16]). Suppose that $\Omega \in L^{s}\left(S^{n-1}\right)(s>$ 1) is homogeneous of degree zero and satisfies the vanishing condition $\int_{S^{n-1}} \Omega\left(x^{\prime}\right) d \sigma\left(x^{\prime}\right)=0$. If $b \in \mathrm{BMO}\left(\mathbb{R}^{n}\right)$, then $\left[b, T_{\Omega}\right]$ is bounded on $L^{p}(w)$ if the index set $\{p, q, s\}$ satisfies one of the following conditions:
(a) $s^{\prime} \leq p<\infty, p \neq 1$ and $w \in A_{p / s^{\prime}}$;
(b) $1 \leq p \leq s, p \neq \infty$ and $w^{1-p^{\prime}} \in A_{p^{\prime} / s^{\prime}}$;
(c) $1 \leq p<\infty$ and $w^{s^{\prime}} \in A_{p}$.

Theorem 23 (see [2]). If $\Omega \in L^{\infty}\left(S^{n-1}\right)$ is homogeneous of degree zero and satisfies the moment condition $\int_{S^{n-1}} \theta \Omega(\theta) d \theta=$ $0, w \in A_{p}, 1<p<\infty, \nabla A \in \mathrm{BMO}$, then we have

$$
\left\|\widetilde{T}_{\Omega}^{A} f\right\|_{L^{p}(w)} \leq C\|\Omega\|_{\infty}\|\nabla A\|_{*}\|f\|_{L^{p}(w)} .
$$

Lemma 24 (see [15]). The following are true:

(1) If $w \in A_{p}$ for some $1 \leq p<\infty$, then $w \in \Delta_{2}$. More precisely, for all $\lambda>1$ we have

$$
w(\lambda Q) \leq C \lambda^{n p} w(Q) .
$$

(2) If $w \in A_{p}$ for some $1 \leq p<\infty$, then there exist $C>0$ and $\delta>0$ such that for any cube $Q$ and a measurable set $S \subset Q$,

$$
\frac{w(S)}{w(Q)} \leq C\left(\frac{|S|}{|Q|}\right)^{\delta}
$$

Lemma 25 (see [17]). Let $w \in A_{\infty}$. Then the norm of $\mathrm{BMO}(w)$ is equivalent to the norm of $\mathrm{BMO}\left(\mathbb{R}^{n}\right)$, where

$$
\begin{aligned}
& \operatorname{BMO}(w)=\left\{b:\|b\|_{*, w}\right. \\
& \left.=\sup _{\mathrm{Q}} \frac{1}{w(Q)} \int_{Q}\left|b(x)-m_{\mathrm{Q}, w} b\right| w(x) d x\right\}, \\
& m_{\mathrm{Q}, w} b=\frac{1}{w(Q)} \int_{\mathrm{Q}} b(x) w(x) d x .
\end{aligned}
$$

\section{Proofs of the Main Results}

Before proving Theorem 8 , we give a pointwise estimate of $T_{\Omega, \alpha}^{A} f(x)$ at first. Set

$$
\bar{T}_{\Omega, \alpha+\beta} f(x)=\int_{\mathbb{R}^{n}} \frac{|\Omega(x-y)|}{|x-y|^{n-\alpha-\beta}}|f(y)| d y,
$$

$$
0<\alpha+\beta<n
$$

where $\Omega \in L^{s}\left(S^{n-1}\right)(s>1)$ is homogeneous of degree zero in $\mathbb{R}^{n}$. Then we have the following estimate.

Theorem 26. If $\alpha \geq 0,0<\alpha+\beta<n, D^{\gamma} A \in \dot{\Lambda}_{\beta}(|\gamma|=m-1)$, then there exists a constant $C$ independent of $f$ such that

$$
\left|T_{\Omega, \alpha}^{A} f(x)\right| \leq C\left(\sum_{|\gamma|=m-1}\left\|D^{\gamma} A\right\|_{\dot{\Lambda}_{\beta}}\right) \bar{T}_{\Omega, \alpha+\beta} f(x) .
$$

Proof. For fixed $x \in \mathbb{R}^{n}, r>0$, let $Q$ be a cube with center at $x$ and diameter $r$. Denote $Q_{k}=2^{k} Q$ and set

$$
A_{\mathrm{Q}_{k}}(y)=A(y)-\sum_{|\gamma|=m-1} \frac{1}{\gamma !} m_{\mathrm{Q}_{k}}\left(D^{\gamma} A\right) y^{\gamma},
$$

where $m_{Q_{k}} f$ is the average of $f$ on $Q_{k}$. Then we have, when $|\gamma|=m-1$,

$$
D^{\gamma} A_{\mathrm{Q}_{k}}(y)=D^{\gamma} A(y)-m_{\mathrm{Q}_{k}}\left(D^{\gamma} A\right)
$$

and it is proved in [1] that

$$
R_{m}(A ; x, y)=R_{m}\left(A_{\mathrm{Q}_{k}} ; x, y\right) .
$$

Hence,

$$
\begin{aligned}
& \left|T_{\Omega, \alpha}^{A} f(x)\right| \\
& \leq \sum_{k=-\infty}^{\infty} \int_{2^{k-1} r \leq|x-y|<2^{k} r} \frac{\left|R_{m}\left(A_{Q_{k}} ; x, y\right)\right|}{|x-y|^{m-1}} \frac{|\Omega(x-y)|}{|x-y|^{n-\alpha}}|f(y)| d y \\
& :=\sum_{k=-\infty}^{\infty} T_{k} .
\end{aligned}
$$

By Lemma 15 we get

$$
\begin{aligned}
& \left|R_{m}\left(A_{\mathrm{Q}_{k}} ; x, y\right)\right| \\
& \leq\left|R_{m-1}\left(A_{\mathrm{Q}_{k}} ; x, y\right)\right| \\
& \quad+C \sum_{|\gamma|=m-1}\left|D^{\gamma} A_{\mathrm{Q}_{k}}(y)\right||x-y|^{m-1} \\
& \leq C|x-y|^{m-1} \sum_{|\gamma|=m-1}\left(\frac{1}{\left|I_{x}^{y}\right|} \int_{I_{x}^{y}}\left|D^{\gamma} A_{\mathrm{Q}_{k}}(z)\right|^{l} d z\right)^{1 / l} \\
& \quad+C|x-y|^{m-1} \sum_{|\gamma|=m-1}\left|D^{\gamma} A_{\mathrm{Q}_{k}}(y)\right| .
\end{aligned}
$$


Note that, if $|x-y|<2^{k} r$, then $I_{x}^{y} \subset 5 n Q_{k}$. By Lemmas 16 and 17 we have, when $|\gamma|=m-1$,

$$
\begin{aligned}
\left(\frac{1}{\left|I_{x}^{y}\right|} \int_{I_{x}^{y}}\left|D^{\gamma} A_{\mathrm{Q}_{k}}(z)\right|^{l} d z\right)^{1 / l} & \\
= & \left(\frac{1}{\left|I_{x}^{y}\right|} \int_{I_{x}^{y}}\left|D^{\gamma} A(z)-m_{\mathrm{Q}_{k}}\left(D^{\gamma} A\right)\right|^{l} d z\right)^{1 / l} \\
\leq & \left(\frac{1}{\left|I_{x}^{y}\right|} \int_{I_{x}^{y}}\left|D^{\gamma} A(z)-m_{I_{x}^{y}}\left(D^{\gamma} A\right)\right|^{l} d z\right)^{1 / l} \\
& +\left|m_{I_{x}^{y}}\left(D^{\gamma} A\right)-m_{5 n \mathrm{Q}_{k}}\left(D^{\gamma} A\right)\right| \\
& +\left|m_{5 n \mathrm{Q}_{k}}\left(D^{\gamma} A\right)-m_{\mathrm{Q}_{k}}\left(D^{\gamma} A\right)\right| \\
\leq & C\left|Q_{k}\right|^{\beta / n}\left\|D^{\gamma} A\right\|_{\dot{\Lambda}_{\beta}} \leq C\left(2^{k} r\right)^{\beta}\left\|D^{\gamma} A\right\|_{\dot{\Lambda}_{\beta}} .
\end{aligned}
$$

It is obvious that when $|\gamma|=m-1$,

$$
\begin{aligned}
\left|D^{\gamma} A_{Q_{k}}(y)\right| & =\left|D^{\gamma} A(y)-m_{Q_{k}}\left(D^{\gamma} A\right)\right| \\
& \leq C\left|Q_{k}\right|^{\beta / n}\left\|D^{\gamma} A\right\|_{\Lambda_{\beta}} \\
& \leq C\left(2^{k} r\right)^{\beta}\left\|D^{\gamma} A\right\|_{\Lambda_{\beta}} .
\end{aligned}
$$

Thus,

$$
\begin{aligned}
& \left|R_{m}\left(A_{\mathrm{Q}_{k}} ; x, y\right)\right| \\
& \quad \leq C|x-y|^{m-1}\left(2^{k} r\right)^{\beta} \sum_{|\gamma|=m-1}\left\|D^{\gamma} A\right\|_{\dot{\Lambda}_{\beta}} .
\end{aligned}
$$

Therefore,

$$
\begin{aligned}
T_{k} & \leq C \sum_{|\gamma|=m-1}\left\|D^{\gamma} A\right\|_{\dot{\Lambda}_{\beta}} \\
& \cdot \int_{2^{k-1} r \leq|x-y|<2^{k} r} \frac{\left(2^{k} r\right)^{\beta}}{|x-y|^{n-\alpha}}|\Omega(x-y)||f(y)| d y \\
& \leq C \sum_{|\gamma|=m-1}\left\|D^{\gamma} A\right\|_{\dot{\Lambda}_{\beta}} \\
& \cdot \int_{2^{k-1} r \leq|x-y|<2^{k} r} \frac{|\Omega(x-y)|}{|x-y|^{n-\alpha-\beta}}|f(y)| d y .
\end{aligned}
$$

It follows that

$$
\begin{aligned}
& \left|T_{\Omega, \alpha}^{A} f(x)\right| \leq \sum_{k=-\infty}^{\infty}\left(C \sum_{|\gamma|=m-1}\left\|D^{\gamma} A\right\|_{\dot{\Lambda}_{\beta}}\right. \\
& \left.\cdot \int_{2^{k-1} r \leq|x-y|<2^{k} r} \frac{|\Omega(x-y)|}{|x-y|^{n-\alpha-\beta}}|f(y)| d y\right) \\
& \leq C \sum_{|\gamma|=m-1}\left\|D^{\gamma} A\right\|_{\dot{\Lambda}_{\beta}} \\
& \cdot \sum_{k=-\infty}^{\infty} \int_{2^{k-1} r \leq|x-y|<2^{k} r} \frac{|\Omega(x-y)|}{|x-y|^{n-\alpha-\beta}}|f(y)| d y \\
& =C \sum_{|\gamma|=m-1}\left\|D^{\gamma} A\right\|_{\dot{\Lambda}_{\beta}} \int_{\mathbb{R}^{n}} \frac{|\Omega(x-y)|}{|x-y|^{n-\alpha-\beta}}|f(y)| d y \\
& =C \sum_{|\gamma|=m-1}\left\|D^{\gamma} A\right\|_{\dot{\Lambda}_{\beta}} \bar{T}_{\Omega, \alpha+\beta} f(x) .
\end{aligned}
$$

Thus, we finish the proof of Theorem 26.

The following theorem is a key theorem in proving (18) of Theorem 8.

Theorem 27. Under the same assumptions of Theorem 8, $\bar{T}_{\Omega, \alpha+\beta}$ is bounded from $L^{p, \kappa}\left(w^{p}, w^{q}\right)$ to $L^{q, \kappa q / p}\left(w^{q}\right)$.

Proof. Fix a ball $B\left(x_{0}, r_{B}\right)$, we decompose $f=f_{1}+f_{2}$ with $f_{1}=f \chi_{2 B}$. Then we have

$$
\begin{aligned}
& \left(\frac{1}{w^{q}(B)^{\kappa q / p}} \int_{B}\left|\bar{T}_{\Omega, \alpha+\beta} f(x)\right|^{q} w^{q}(x) d x\right)^{1 / q} \\
& \leq \frac{1}{w^{q}(B)^{\kappa / p}}\left(\int_{B}\left|\bar{T}_{\Omega, \alpha+\beta} f_{1}(x)\right|^{q} w^{q}(x) d x\right)^{1 / q} \\
& \quad+\frac{1}{w^{q}(B)^{\kappa / p}}\left(\int_{B}\left|\bar{T}_{\Omega, \alpha+\beta} f_{2}(x)\right|^{q} w^{q}(x) d x\right)^{1 / q} \\
& :=J_{1}+J_{2} .
\end{aligned}
$$

We estimate $J_{1}$ at first. By Remark 7(a) we know that $w^{q} \in \Delta_{2}$. Then by Theorem 18(a) and the fact that $w^{q} \in \Delta_{2}$ we get,

$$
\begin{aligned}
J_{1} & \leq \frac{1}{w^{q}(B)^{\kappa / p}}\left\|\bar{T}_{\Omega, \alpha+\beta} f_{1}\right\|_{L^{q}\left(w^{q}\right)} \\
& \leq \frac{C}{w^{q}(B)^{\kappa / p}}\left\|f_{1}\right\|_{L^{p}\left(w^{p}\right)} \\
& =\frac{C}{w^{q}(B)^{\kappa / p}}\left(\int_{2 B}|f(x)|^{p} w(x)^{p} d x\right)^{1 / p} \\
& \leq C\|f\|_{L^{p, \kappa}\left(w^{p}, w^{q}\right)} \frac{w^{q}(2 B)^{\kappa / p}}{w^{q}(B)^{\kappa / p}} \leq C\|f\|_{L^{p, \kappa}\left(w^{p}, w^{q}\right)} .
\end{aligned}
$$


Now we consider the term $J_{2}$. By Hölder's inequality, we have

$$
\begin{aligned}
& \left|\bar{T}_{\Omega, \alpha+\beta} f_{2}(x)\right|=\sum_{j=1}^{\infty} \int_{2^{j+1} B \backslash 2^{j} B} \frac{|\Omega(x-y)|}{|x-y|^{n-\alpha-\beta}}|f(y)| d y \\
& \leq C \sum_{j=1}^{\infty}\left(\int_{2^{j+1} B}|\Omega(x-y)|^{s} d y\right)^{1 / s} \\
& \quad \cdot\left(\int_{2^{j+1} B \backslash 2^{j} B} \frac{|f(y)|^{s^{\prime}}}{|x-y|^{(n-\alpha-\beta) s^{\prime}}} d y\right)^{1 / s^{\prime}} \\
& :=C \sum_{j=1}^{\infty}\left(I_{1 j} I_{2 j}\right) .
\end{aligned}
$$

We will estimate $I_{1 j}, I_{2 j}$, respectively. Let $z=x-y$; then for $x \in B, y \in 2^{j+1} B$, we have $z \in 2^{j+2} B$. Noticing that $\Omega$ is homogeneous of degree zero and $\Omega \in L^{s}\left(S^{n-1}\right)$, then we have

$$
\begin{aligned}
I_{1 j} & =\left(\int_{2^{j+2} B}|\Omega(z)|^{s} d z\right)^{1 / s} \\
& =\left(\int_{0}^{2^{j+2} r_{B}} \int_{S^{n-1}}\left|\Omega\left(z^{\prime}\right)\right|^{s} d z^{\prime} r^{n-1} d r\right)^{1 / s} \\
& =C\|\Omega\|_{L^{s}\left(S^{n-1}\right)}\left|2^{j+2} B\right|^{1 / s},
\end{aligned}
$$

where $z^{\prime}=z /|z|$. For $x \in B, y \in(2 B)^{c}$, we have $|x-y| \sim$ $\left|x_{0}-y\right|$. Thus,

$$
I_{2 j} \leq \frac{C}{\left|2^{j+1} B\right|^{1-(\alpha+\beta) / n}}\left(\int_{2^{j+1} B}|f(y)|^{s^{\prime}} d y\right)^{1 / s^{\prime}} .
$$

By Hölder's inequality and $w^{s^{\prime}} \in A\left(p / s^{\prime}, q / s^{\prime}\right)$, we get

$$
\begin{aligned}
& \left(\int_{2^{j+1} B}|f(y)|^{s^{\prime}} d y\right)^{1 / s^{\prime}} \\
& \leq C\left(\int_{2^{j+1} B}|f(y)|^{p} w(y)^{p} d y\right)^{1 / p} \\
& \cdot\left(\int_{2^{j+1} B} w(y)^{-p s^{\prime} /\left(p-s^{\prime}\right)} d y\right)^{\left(p-s^{\prime}\right) / p s^{\prime}} \\
& \leq C\|f\|_{L^{p, \kappa}\left(w^{p}, w^{q}\right)} w^{q}\left(2^{j+1} B\right)^{\kappa / p} \\
& \quad \cdot\left(\int_{2^{j+1} B} w(y)^{-p s^{\prime} /\left(p-s^{\prime}\right)} d y\right)^{\left(p-s^{\prime}\right) / p s^{\prime}} \\
& \quad \leq C\|f\|_{L^{p, \kappa}\left(w^{p}, w^{q}\right)} w^{q}\left(2^{j+1} B\right)^{\kappa / p} \\
& . \\
& \quad \frac{\left|2^{j+1} B\right|^{\left(p q-s^{\prime} q+s^{\prime} p\right) / p q s^{\prime}}}{w q\left(2^{j+1} B\right)^{1 / q}} .
\end{aligned}
$$

Thus,

$$
\begin{aligned}
& \left|\bar{T}_{\Omega, \alpha+\beta} f_{2}(x)\right| \leq C \sum_{j=1}^{\infty}\left(I_{1 j} I_{2 j}\right) \\
& \quad \leq C \sum_{j=1}^{\infty}\|f\|_{L^{p, \kappa}\left(w^{p}, w^{q}\right)} \frac{1}{w^{q}\left(2^{j+1} B\right)^{1 / q-\kappa / p}} .
\end{aligned}
$$

So we get

$$
J_{2} \leq C\|f\|_{L^{p, \kappa}\left(w^{p}, w^{q}\right)} \sum_{j=1}^{\infty} \frac{w^{q}(B)^{1 / q-\kappa / p}}{w^{q}\left(2^{j+1} B\right)^{1 / q-\kappa / p}} .
$$

We know from Remark 7(a) and Lemma 19 that $w^{q}$ satisfies inequality (33), so the above series converges since the reverse doubling constant is larger than one. Hence,

$$
J_{2} \leq C\|f\|_{L^{p, \kappa}\left(w^{p}, w^{q}\right)} .
$$

Therefore, the proof of Theorem 27 is completed.

Remark 28. It is worth noting that Theorem 27 is essentially verifying the multilinear fractional operator $T_{\Omega, \alpha}$ is bounded on weighted Morrey spaces.

Now we are in the position of proving Theorem 8 .

We will obtain (18) immediately in combination of Theorems 26 and 27.

Then let us turn to prove (19).

Set

$$
\begin{array}{r}
\bar{T}_{\Omega, \alpha}^{A} f(x) \\
=\int_{\mathbb{R}^{n}} \frac{|\Omega(x-y)|}{|x-y|^{n-\alpha+m-1}}\left|R_{m}(A ; x, y)\right||f(y)| d y, \\
\quad 0 \leq \alpha<n,
\end{array}
$$

where $\Omega \in L^{s}\left(S^{n-1}\right)(s>1)$ is homogeneous of degree zero in $\mathbb{R}^{n}$. It is easy to see inequality (18) also holds for $\bar{T}_{\Omega, \alpha}^{A}$. On the other hand, for any $r>0$, we have

$$
\begin{aligned}
& \bar{T}_{\Omega, \alpha}^{A} f(x) \\
& \quad \geq \int_{|x-y|<r} \frac{|\Omega(x-y)|}{|x-y|^{n-\alpha+m-1}}\left|R_{m}(A ; x, y)\right||f(y)| d y \\
& \quad \geq \frac{1}{r^{n-\alpha+m-1}} \\
& \quad \cdot \int_{|x-y|<r}|\Omega(x-y)|\left|R_{m}(A ; x, y)\right||f(y)| d y .
\end{aligned}
$$

Taking the supremum for $r>0$ on the inequality above, we get

$$
\bar{T}_{\Omega, \alpha}^{A} f(x) \geq M_{\Omega, \alpha}^{A} f(x) .
$$

Thus, we can immediately obtain (19) from (65) and (18). 
Similarly as before, we give the following theorem at first before proving Theorem 9, since it plays an important role in the proof of Theorem 9. Set

$$
\bar{T}_{\Omega, \beta} f(x)=\int_{\mathbb{R}^{n}} \frac{|\Omega(x-y)|}{|x-y|^{n-\beta}}|f(y)| d y,
$$

where $\Omega \in L^{s}\left(S^{n-1}\right)(s>1)$ is homogeneous of degree zero in $\mathbb{R}^{n}$.

Theorem 29. Under the assumptions of Theorem $9, \bar{T}_{\Omega, \beta}$ is bounded from $L^{p, \kappa}\left(w^{p}, w^{q}\right)$ to $L^{q, \kappa q / p}\left(w^{q}\right)$.

The proof of Theorem 29 can be treated as that of Theorem 27 with only slight modifications; we omit its proof here.

Now, let us prove Theorem 9. It is not difficult to see that (20) can be easily obtained from Theorems 26 and 29. Then we can immediately arrive at (21) from (65) and (20).

From now on, we are in the place of showing Theorem 10. We prove (22) at first. Fixing any cube $Q$ with center at $x$ and diameter $r$, denote $\bar{Q}=2 Q$ and set

$$
A_{\overline{\mathrm{Q}}}(y)=A(y)-\sum_{|\gamma|=m-1} \frac{1}{\gamma !} m_{\overline{\mathrm{Q}}}\left(D^{\gamma} A\right) y^{\gamma} .
$$

Noticing that equality (67) is the special case of equality (44) when $k=1$. Thus, equalities (45) and (46) also hold for $A_{\bar{Q}}(y)$. We decompose $f$ as $f=f \chi_{\bar{Q}}+f \chi_{(\bar{Q})^{c}}:=f_{1}+f_{2}$. Then we have

$$
\begin{aligned}
& \frac{1}{w^{q}(Q)^{\kappa / p}}\left(\int_{Q}\left|T_{\Omega, \alpha}^{A} f(y)\right|^{q} w(y)^{q} d y\right)^{1 / q} \\
& \leq \frac{1}{w^{q}(Q)^{\kappa / p}}\left(\int_{Q}\left|T_{\Omega, \alpha}^{A} f_{1}(y)\right|^{q} w(y)^{q} d y\right)^{1 / q} \\
& \quad+\frac{1}{w^{q}(Q)^{\kappa / p}}\left(\int_{Q}\left|T_{\Omega, \alpha}^{A} f_{2}(y)\right|^{q} w(y)^{q} d y\right)^{1 / q} \\
& :=I+I I .
\end{aligned}
$$

By Theorem 20(a) and Remark 7(a) that $w^{q} \in \Delta_{2}$, we have

$$
\begin{aligned}
I & \leq \frac{C}{w^{q}(Q)^{\kappa / p}} \\
& \cdot \sum_{|\gamma|=m-1}\left\|D^{\gamma} A\right\|_{*}\left(\int_{\bar{Q}}|f(y)|^{p} w(y)^{p} d y\right)^{1 / p} \\
& =C \sum_{|\gamma|=m-1}\left\|D^{\gamma} A\right\|_{*}\|f\|_{L^{p, \kappa}\left(w^{p}, w^{q}\right)}\left(\frac{w^{q}(\bar{Q})}{w^{q}(Q)}\right)^{\kappa / p} \\
& \leq C \sum_{|\gamma|=m-1}\left\|D^{\gamma} A\right\|_{*}\|f\|_{L^{p, \kappa}\left(w^{p}, w^{q}\right)} .
\end{aligned}
$$

Next, we consider the term $T_{\Omega, \alpha}^{A} f_{2}(y)$ contained in II. By Lemma 15 and equality (45), (46), we have

$$
\begin{aligned}
& \left|T_{\Omega, \alpha}^{A} f_{2}(y)\right| \\
& \quad \leq \int_{(\overline{\mathrm{Q}})^{c}} \frac{\left|R_{m}\left(A_{\overline{\mathrm{Q}}} ; y, z\right)\right|}{|y-z|^{m-1}} \frac{|\Omega(y-z)|}{|y-z|^{n-\alpha}}|f(z)| d z \\
& \quad \leq C \int_{(\overline{\mathrm{Q}})^{c}} \sum_{|\gamma|=m-1}\left(\frac{1}{\left|I_{y}^{z}\right|} \int_{I_{y}^{z}}\left|D^{\gamma} A_{\overline{\mathrm{Q}}}(t)\right|^{l} d t\right)^{1 / l} \\
& \cdot|\Omega(y-z)| \frac{|f(z)|}{|y-z|^{n-\alpha}} d z \\
& \quad+C \int_{(\overline{\mathrm{Q}})^{c}} \sum_{|y|=m-1}\left|D^{\gamma} A(z)-m_{\overline{\mathrm{Q}}}\left(D^{\gamma} A\right)\right||\Omega(y-z)| \\
& \cdot \frac{|f(z)|}{|y-z|^{n-\alpha}} d z:=I I_{1}+I I_{2} .
\end{aligned}
$$

We estimate $I I_{1}$ and $I I_{2}$, respectively. By Lemma 21(a) and (b), Hölder's inequality, and $w^{s^{\prime}} \in A\left(p / s^{\prime}, q / s^{\prime}\right)$, we get

$$
\begin{aligned}
& I I_{1} \leq C \sum_{|\gamma|=m-1}\left(\frac{1}{\left|I_{y}^{z}\right|} \int_{I_{y}^{z}}\left|D^{\gamma} A(t)-m_{\overline{\mathrm{Q}}}\left(D^{\gamma} A\right)\right|^{l} d t\right)^{1 / l} \\
& \quad \sum_{j=1}^{\infty} \int_{\left.2^{j+1} \mathrm{Q}\right|^{j} \mathrm{Q}}|\Omega(y-z)| \frac{|f(z)|}{|y-z|^{n-\alpha}} d z \\
& \quad \leq C \sum_{|\gamma|=m-1}\left[\left(\frac{1}{\left|I_{y}^{z}\right|}\right.\right. \\
& \left.\cdot \int_{I_{y}^{z}}\left|D^{\gamma} A(t)-m_{I_{y}^{z}}\left(D^{\gamma} A\right)\right|^{l} d t\right)^{1 / l}+\mid m_{I_{y}^{z}}\left(D^{\gamma} A\right) \\
& \left.-m_{5 n \overline{\mathrm{Q}}}\left(D^{\gamma} A\right)|+| m_{5 n \overline{\mathrm{Q}}}\left(D^{\gamma} A\right)-m_{\overline{\mathrm{Q}}}\left(D^{\gamma} A\right) \mid\right]
\end{aligned}
$$

$$
\begin{aligned}
& \cdot \sum_{j=1}^{\infty}\left(\int_{2^{j+1} Q}|\Omega(y-z)|^{s} d z\right)^{1 / s} \\
& \cdot\left(\int_{2^{j+1} Q \mid 2^{j} Q} \frac{|f(z)|^{s^{\prime}}}{|y-z|^{(n-\alpha) s^{\prime}}} d z\right)^{1 / s^{\prime}} \\
& \leq C \sum_{|\gamma|=m-1}\left\|D^{\gamma} A\right\|_{*}\|\Omega\|_{L^{s}\left(S^{n-1}\right)} \\
& \cdot \sum_{j=1}^{\infty} \frac{\left|2^{j+2} Q\right|^{1 / s}}{\left|2^{j} Q\right|^{1-\alpha / n}}\left(\int_{2^{j+1} Q}|f(z)|^{s^{\prime}} d z\right)^{1 / s^{\prime}}
\end{aligned}
$$




$$
\begin{array}{lrl}
\leq C \sum_{|\gamma|=m-1}\left\|D^{\gamma} A\right\|_{*} \sum_{j=1}^{\infty} \frac{\left|2^{j+2} Q\right|^{1 / s}}{\left|2^{j} Q\right|^{1-\alpha / n}}\left(\int_{2^{j+1} Q}|f(z)|^{p}\right. & \text { For } y \in Q, z \in(\bar{Q})^{c} \text {, we have }|y-z| \sim|x-z| \text {, so we obtain } \\
\left.\cdot w(z)^{p} d z\right)^{1 / p} & I I_{2} \leq C \sum_{j=1}^{\infty} \int_{2^{j+1} Q \mid 2^{j} Q} \sum_{|\gamma|=m-1}\left|D^{\gamma} A(z)-m_{2^{j+1} Q}\left(D^{\gamma} A\right)\right| \\
\cdot\left(\int_{2^{j+1} Q} w(z)^{-p s^{\prime} /\left(p-s^{\prime}\right)} d z\right)^{\left(p-s^{\prime}\right) / p s^{\prime}} & \cdot|\Omega(y-z)| \frac{|f(z)|}{|y-z|^{n-\alpha}} d z \\
\leq C \sum_{|\gamma|=m-1}\left\|D^{\gamma} A\right\|_{*}\|f\|_{L^{p, \kappa}\left(w^{p}, w^{q}\right)} & +C \sum_{j=1}^{\infty} \int_{2^{j+1} Q \mid 2^{j} Q} \sum_{|\gamma|=m-1}\left|m_{2^{j+1} Q}\left(D^{\gamma} A\right)-m_{\bar{Q}}\left(D^{\gamma} A\right)\right| \\
\cdot \sum_{j=1}^{\infty} \frac{1}{w^{q}\left(2^{j+1} Q\right)^{1 / q-\kappa / p}} . & \cdot|\Omega(y-z)| \frac{|f(z)|}{|y-z|^{n-\alpha}} d z:=I I_{21}+I I_{22} .
\end{array}
$$

(71) By Hölder’s inequality, we get

$$
\begin{aligned}
I I_{21} \leq & C \sum_{j=1}^{\infty} \frac{1}{\left|2^{j} Q\right|^{1-\frac{\alpha}{n}}} \int_{2^{j+1} Q} \sum_{|\gamma|=m-1}\left|D^{\gamma} A(z)-m_{2^{j+1} Q}\left(D^{\gamma} A\right)\right||\Omega(y-z)||f(z)| d z \\
\leq & C \sum_{j=1}^{\infty} \frac{1}{\left|2^{j} Q\right|^{1-\frac{\alpha}{n}}}\left(\int_{2^{j+1} Q} \sum_{|\gamma|=m-1}\left|D^{\gamma} A(z)-m_{2^{j+1} Q}\left(D^{\gamma} A\right)\right|^{s^{\prime}}|f(z)|^{s^{\prime}} d z\right)^{1 / s^{\prime}}\left(\int_{2^{j+1} Q}|\Omega(y-z)|^{s} d z\right)^{1 / s} \\
\leq & C\|\Omega\|_{L^{s}\left(S^{n-1}\right)} \sum_{j=1}^{\infty} \frac{\left|2^{j+2} Q\right|^{1 / s}}{\left|2^{j} Q\right|^{1-\frac{\alpha}{n}}}\left(\int_{2^{j+1} Q} \sum_{|\gamma|=m-1}\left|D^{\gamma} A(z)-m_{2^{j+1} Q}\left(D^{\gamma} A\right)\right|^{s^{\prime}}|f(z)|^{s^{\prime}} d z\right)^{1 / s^{\prime}} \\
\leq & C\|\Omega\|_{L^{s}\left(S^{n-1}\right)} \sum_{j=1}^{\infty} \frac{\left|2^{j^{j+2}} Q\right|^{1 / s}}{\left|2^{j} Q\right|^{1-\frac{\alpha}{n}}}\left(\int_{2^{j+1} Q}|f(z)|^{p} w(z)^{p} d z\right)^{1 / p} \\
& \cdot\left(\int_{2^{j+1} Q} \sum_{|\gamma|=m-1}\left|D^{\gamma} A(z)-m_{2^{j+1} Q}\left(D^{\gamma} A\right)\right|^{p s^{\prime} /\left(p-s^{\prime}\right)} w(z)^{-p s^{\prime} /\left(p-s^{\prime}\right)} d z\right)^{\left(p-s^{\prime}\right) / p s^{\prime}}
\end{aligned}
$$

We estimate the part containing the function $D^{\gamma} A$ as follows:

$$
\begin{aligned}
& \left(\int_{2^{j+1} Q} \sum_{|\gamma|=m-1}\left|D^{\gamma} A(z)-m_{2^{j+1} Q}\left(D^{\gamma} A\right)\right|^{p s^{\prime} /\left(p-s^{\prime}\right)} w(z)^{-p s^{\prime} /\left(p-s^{\prime}\right)} d z\right)^{\left(p-s^{\prime}\right) / p s^{\prime}} \\
& \leq C\left(\int_{2^{j+1}} \sum_{|\gamma|=m-1}\left|D^{\gamma} A(z)-m_{2^{j+1} \mathrm{Q}, w^{-p s^{\prime} /\left(p-s^{\prime}\right)}}\left(D^{\gamma} A\right)\right|^{p s^{\prime} /\left(p-s^{\prime}\right)} w(z)^{-p s^{\prime} /\left(p-s^{\prime}\right)} d z\right)^{\left(p-s^{\prime}\right) / p s^{\prime}} \\
& +\sum_{|\gamma|=m-1}\left|m_{2^{j+1} \mathrm{Q}, w^{-p s^{\prime} /\left(p-s^{\prime}\right)}}\left(D^{\gamma} A\right)-m_{2^{j+1} \mathrm{Q}}\left(D^{\gamma} A\right)\right| w^{-p s^{\prime} /\left(p-s^{\prime}\right)}\left(2^{j+1} \mathrm{Q}\right)^{\left(p-s^{\prime}\right) / p s^{\prime}}:=I I I+I V .
\end{aligned}
$$

For the term III, since $w^{s^{\prime}} \in A\left(p / s^{\prime}, q / s^{\prime}\right)$, we then have $w^{-p s^{\prime} /\left(p-s^{\prime}\right)} \in A_{t^{\prime}} \subset A_{\infty}$ by Remark 7(b). Thus, by Lemma 25 that the norm of $\mathrm{BMO}\left(w^{-p s^{\prime} /\left(p-s^{\prime}\right)}\right)$ is equivalent to the norm of $\operatorname{BMO}\left(\mathbb{R}^{n}\right)$ and $w^{s^{\prime}} \in A\left(p / s^{\prime}, q / s^{\prime}\right)$, we have 


$$
\begin{aligned}
I I I & \leq C \sum_{|\gamma|=m-1}\left\|D^{\gamma} A\right\|_{*} w^{-p s^{\prime} /\left(p-s^{\prime}\right)}\left(2^{j+1} Q\right)^{\left(p-s^{\prime}\right) / p s^{\prime}} \\
& =C \sum_{|\gamma|=m-1}\left\|D^{\gamma} A\right\|_{*} \frac{\left|2^{j+1} Q\right|^{\left(p-s^{\prime}\right) / p s^{\prime}+1 / q}}{w^{q}\left(2^{j+1} Q\right)^{1 / q}} .
\end{aligned}
$$

For the term $I V$, by Lemma 21(a), there exist $C_{1}, C_{2}>0$ such that for any cube $Q$ and $s>0$,

$$
\begin{aligned}
& \left|\left\{t \in 2^{j+1} Q: \sum_{|\gamma|=m-1}\left|D^{\gamma} A(t)-m_{2^{j+1} Q}\left(D^{\gamma} A\right)\right|>s\right\}\right| \\
& \quad \leq C_{1}\left|2^{j+1} Q\right| e^{-C_{2} s /\left(\sum_{|\gamma|=m-1}\left\|D^{\gamma} A\right\|_{*}\right)},
\end{aligned}
$$

since $\sum_{|\gamma|=m-1}\left(D^{\gamma} A\right) \in \mathrm{BMO}$. Then by Lemma 24(2), we have

$$
\begin{gathered}
w\left(\left\{t \in 2^{j+1} Q: \sum_{|\gamma|=m-1}\left|D^{\gamma} A(t)-m_{2^{j+1} Q}\left(D^{\gamma} A\right)\right|\right.\right. \\
>s\}) \leq C w\left(2^{j+1} Q\right) e^{-C_{2} s \delta /\left(\sum_{|\gamma|=m-1}\left\|D^{\gamma} A\right\|_{*}\right)},
\end{gathered}
$$

for some $\delta>0$. Hence it implies

$$
\begin{aligned}
& \sum_{|\gamma|=m-1}\left|m_{2^{j+1} \mathrm{Q}, w^{-p s^{\prime} /\left(p-s^{\prime}\right)}}\left(D^{\gamma} A\right)-m_{2^{j+1} \mathrm{Q}}\left(D^{\gamma} A\right)\right| \\
& \leq \frac{1}{w^{-p s^{\prime} /\left(p-s^{\prime}\right)}\left(2^{j+1} Q\right)} \int_{2^{j+1} Q} \sum_{|\gamma|=m-1} \mid D^{\gamma} A(t) \\
& -m_{2^{j+1} \mathrm{Q}}\left(D^{\gamma} A\right) \mid w^{-p s^{\prime} /\left(p-s^{\prime}\right)}(t) d t \\
& =\frac{C}{w^{-p s^{\prime} /\left(p-s^{\prime}\right)}\left(2^{j+1} Q\right)} \int_{0}^{\infty} w^{-p s^{\prime} /\left(p-s^{\prime}\right)}(\{t \\
& \in 2^{j+1} Q: \sum_{|\gamma|=m-1}\left|D^{\gamma} A(t)-m_{2^{j+1} Q}\left(D^{\gamma} A\right)\right| \\
& >s\}) d s \leq \frac{C}{w^{-p s^{\prime} /\left(p-s^{\prime}\right)}\left(2^{j+1} Q\right)} \\
& \cdot \int_{0}^{\infty} w^{-p s^{\prime} /\left(p-s^{\prime}\right)}\left(2^{j+1} Q\right) e^{-C_{2} s \delta /\left(\sum_{|\gamma|=m-1}\left\|D^{\gamma} A\right\|_{*}\right)} d s \\
& =C \sum_{|\gamma|=m-1}\left\|D^{\gamma} A\right\|_{*} .
\end{aligned}
$$

As a result,

$$
\begin{aligned}
I V & \leq C \sum_{|\gamma|=m-1}\left\|D^{\gamma} A\right\|_{*} w^{-p s^{\prime} /\left(p-s^{\prime}\right)}\left(2^{j+1} Q\right)^{\left(p-s^{\prime}\right) / p s^{\prime}} \\
& =C \sum_{|\gamma|=m-1}\left\|D^{\gamma} A\right\|_{*} \frac{\left|2^{j+1} Q\right|^{\left(p-s^{\prime}\right) / p s^{\prime}+1 / q}}{w^{q}\left(2^{j+1} Q\right)^{1 / q}} .
\end{aligned}
$$

Thus,

$$
\begin{aligned}
I I_{21} \leq & C \sum_{|\gamma|=m-1}\left\|D^{\gamma} A\right\|_{*}\|f\|_{L^{p, \kappa}\left(w^{p}, w^{q}\right)} \\
& \cdot \sum_{j=1}^{\infty} \frac{1}{w^{q}\left(2^{j+1} Q\right)^{1 / q-\kappa / p}} .
\end{aligned}
$$

For the term $I I_{22}$, by Lemma 21(c), Hölder's inequality, and $w^{s^{\prime}} \in A\left(p / s^{\prime}, q / s^{\prime}\right)$, we get

$$
\begin{aligned}
& I I_{22} \leq C \sum_{|\gamma|=m-1}\left\|D^{\gamma} A\right\|_{*} \\
& \cdot \sum_{j=1}^{\infty} j \int_{2^{j+1} \mathrm{Q} \mid 2^{j} \mathrm{Q}}|\Omega(y-z)| \frac{|f(z)|}{|y-z|^{n-\alpha}} d z \\
& \leq C \sum_{|\gamma|=m-1}\left\|D^{\gamma} A\right\|_{*}\|\Omega\|_{L^{s}\left(S^{n-1}\right)} \sum_{j=1}^{\infty} j \\
& \cdot \frac{\left|2^{j+2} Q\right|^{1 / s}}{\left|2^{j} Q\right|^{1-\alpha / n}}\left(\int_{2^{j+1} Q}|f(z)|^{s^{\prime}} d z\right)^{1 / s^{\prime}} \\
& \leq C \sum_{|\gamma|=m-1}\left\|D^{\gamma} A\right\|_{*} \sum_{j=1}^{\infty} j \\
& \cdot \frac{\left|2^{j+2} Q\right|^{1 / s}}{\left|2^{j} Q\right|^{1-\alpha / n}}\left(\int_{2^{j+1} Q}|f(z)|^{p} w(z)^{p} d z\right)^{1 / p} \\
& \cdot\left(\int_{2^{j+1} \mathrm{Q}} w(z)^{-p s^{\prime} /\left(p-s^{\prime}\right)} d z\right)^{\left(p-s^{\prime}\right) / p s^{\prime}} \\
& \leq C \sum_{|\gamma|=m-1}\left\|D^{\gamma} A\right\|_{*}\|f\|_{L^{p, \kappa}\left(w^{p}, w^{q}\right)} \\
& \cdot \sum_{j=1}^{\infty} \frac{j}{w^{q}\left(2^{j+1} Q\right)^{1 / q-\kappa / p}} .
\end{aligned}
$$

Hence,

$$
\begin{aligned}
\left|T_{\Omega, \alpha}^{A} f_{2}(y)\right| \leq & C \sum_{|\gamma|=m-1}\left\|D^{\gamma} A\right\|_{*}\|f\|_{L^{p, \kappa}\left(w^{p}, w^{q}\right)} \\
& \cdot \sum_{j=1}^{\infty} \frac{j}{w^{q}\left(2^{j+1} Q\right)^{1 / q-\kappa / p}} .
\end{aligned}
$$

Therefore,

$$
\begin{aligned}
I I \leq & C \sum_{|\gamma|=m-1}\left\|D^{\gamma} A\right\|_{*}\|f\|_{L^{p, \kappa}\left(w^{p}, w^{q}\right)} \\
& \cdot \sum_{j=1}^{\infty} j \frac{w^{q}(Q)^{1 / q-\kappa / p}}{w^{q}\left(2^{j+1} Q\right)^{1 / q-\kappa / p}}
\end{aligned}
$$




$$
\begin{aligned}
& \leq C \sum_{|\gamma|=m-1}\left\|D^{\gamma} A\right\|_{*}\|f\|_{L^{p, \kappa}\left(w^{p}, w^{q}\right)} \sum_{j=1}^{\infty} \frac{j}{\left(D_{1}^{j+1}\right)^{1 / q-\kappa / p}} \\
& \leq C \sum_{|\gamma|=m-1}\left\|D^{\gamma} A\right\|_{*}\|f\|_{L^{p, \kappa}\left(w^{p}, w^{q}\right)}
\end{aligned}
$$

where $D_{1}>1$ is the reverse doubling constant. Consequently,

$$
\begin{aligned}
& \frac{1}{w^{q}(Q)^{\kappa / p}}\left(\int_{Q}\left|T_{\Omega, \alpha}^{A} f(y)\right|^{q} w(y)^{q} d y\right)^{1 / q} \\
& \leq C \sum_{|\gamma|=m-1}\left\|D^{\gamma} A\right\|_{*}\|f\|_{L^{p, \kappa}\left(w^{p}, w^{q}\right)} .
\end{aligned}
$$

Taking supremum over all cubes in $\mathbb{R}^{n}$ on both sides of the above inequality, we complete the proof of (22) of Theorem 10.

It is not difficult to see that inequality (23) is easy to get from (22) and (65).

Proof of Theorem 11. We consider (25) firstly. Let $Q$ be the same as in the proof of (22) and denote $\bar{Q}=2 Q$; we decompose $f$ as $f=f \chi_{\bar{Q}}+f \chi_{(\bar{Q})^{c}}:=f_{1}+f_{2}$. Then we have

$$
\begin{aligned}
& \frac{1}{w(Q)^{\kappa / p}}\left(\int_{Q}\left|\left[A, T_{\Omega}\right] f(y)\right|^{p} w(y) d y\right)^{1 / p} \\
& \leq \frac{1}{w(Q)^{\kappa / p}}\left(\int_{Q}\left|\left[A, T_{\Omega}\right] f_{1}(y)\right|^{p} w(y) d y\right)^{1 / p} \\
& \quad+\frac{1}{w(Q)^{\kappa / p}}\left(\int_{Q}\left|\left[A, T_{\Omega}\right] f_{2}(y)\right|^{p} w(y) d y\right)^{1 / p} \\
& :=I+I I .
\end{aligned}
$$

$$
\begin{aligned}
& \frac{1}{w(Q)^{\kappa / p}}\left(\int_{Q} I I_{1}(y)^{p} w(y) d y\right)^{1 / p} \\
& =C \frac{\|\Omega\|_{L^{s}}}{w(Q)^{\kappa / p}} \sum_{j=1}^{\infty} \frac{\left|2^{j+2} Q\right|^{1 / s}}{\left|2^{j} Q\right|}\left(\int_{Q}\left|A(y)-m_{2^{j+1} \mathrm{Q}, w^{-s^{\prime} /\left(p-s^{\prime}\right)}}(A)\right|^{p}\left(\int_{2^{j+1} \mathrm{Q}}|f(z)|^{s^{\prime}} d z\right)^{p / s^{\prime}} w(y) d y\right)^{1 / p} \\
& \leq C \quad \frac{\|f\|_{L^{p, \kappa}(w)}}{w(Q)} \frac{\infty}{p} \sum_{j=1}^{\infty} \frac{\left|2^{j+2} Q\right|^{1 / s}}{\left|2^{j} Q\right|} w\left(2^{j+1} Q\right)^{\kappa / p} w^{-s^{\prime} /\left(p-s^{\prime}\right)}\left(2^{j+1} Q\right)^{\left(p-s^{\prime}\right) / p s^{\prime}} \cdot\left(\int_{Q}\left|A(y)-m_{2^{j+1} Q, w^{-s^{\prime} /\left(p-s^{\prime}\right)}}(A)\right|^{p} w(y) d y\right)^{1 / p} \\
& \leq C \frac{\|f\|_{L^{p, \kappa}(w)}}{w(Q)^{\kappa / p}} \sum_{j=1}^{\infty} \frac{1}{w\left(2^{j+1} Q\right)^{(1-\kappa) / p}}\left(\int_{Q}\left|A(y)-m_{2^{j+1} Q, w^{-s^{\prime}\left(p-s^{\prime}\right)}}(A)\right|^{p} w(y) d y\right)^{1 / p} .
\end{aligned}
$$

For $\left|\left[A, T_{\Omega}\right] f_{2}(y)\right|$, by Hölder's inequality, we obtain

$$
\begin{aligned}
& \left|\left[A, T_{\Omega}\right] f_{2}(y)\right| \leq \sum_{j=1}^{\infty} \int_{\left.2^{j+1} Q\right|_{2} ^{j} Q} \frac{|\Omega(y-z)|}{|y-z|^{n}}|A(y)-A(z)| \\
& \cdot|f(z)| d z \\
& \leq C \sum_{j=1}^{\infty} \frac{1}{\left|2^{j} Q\right|}\left(\int_{2^{j+1} Q}|\Omega(y-z)|^{s} d z\right)^{1 / s} \\
& \cdot\left(\int_{2^{j+1} Q}|A(y)-A(z)|^{s^{\prime}}|f(z)|^{s^{\prime}} d z\right)^{1 / s^{\prime}} \leq C\|\Omega\|_{L^{s}} \\
& \cdot \sum_{j=1}^{\infty} \frac{\left|2^{j+2} Q\right|^{1 / s}}{\left|2^{j} Q\right|}\left|A(y)-m_{2^{j+1} Q, w^{-s^{\prime}}\left(p-s^{\prime}\right)}(A)\right| \\
& \cdot\left(\int_{2^{j+1} Q}|f(z)|^{s^{\prime}} d z\right)^{1 / s^{\prime}}+C\|\Omega\|_{L^{s}} \\
& \cdot \sum_{j=1}^{\infty} \frac{\left|2^{j+2} Q\right|^{1 / s}}{\left|2^{j} Q\right|}\left(\int_{2^{j+1} Q}\left|m_{2^{j+1} Q, w^{-s^{\prime}}\left(p-s^{\prime}\right)}(A)-A(z)\right|^{s^{\prime}}\right. \\
& \left.\cdot|f(z)|^{s^{\prime}} d z\right)^{1 / s^{\prime}}:=I I_{1}(y)+I I_{2} .
\end{aligned}
$$

Next we estimate $I I_{1}(y)$ and $I I_{2}$, respectively. By Hölder's inequality and $w \in A_{p / s^{\prime}}$, we have 
We estimate the part containing $m_{2^{j+1} \mathrm{Q}, w^{-s^{\prime} /\left(p-s^{\prime}\right)}}(A)$ as follows:

$$
\begin{aligned}
& \left(\int_{\mathrm{Q}}\left|A(y)-m_{2^{j+1} \mathrm{Q}, w^{-s^{\prime} /\left(p-s^{\prime}\right)}}(A)\right|^{p} w(y) d y\right)^{1 / p} \\
& \leq\left(\int_{\mathrm{Q}}\left|A(y)-m_{\mathrm{Q}, w}(A)\right|^{p} w(y) d y\right)^{1 / p} \\
& \quad+\left|m_{\mathrm{Q}, w}(A)-m_{2^{j+1} \mathrm{Q}, w^{-s^{\prime} /\left(p-s^{\prime}\right)}}(A)\right| w(Q)^{1 / p} \\
& :=I I I+I V .
\end{aligned}
$$

For the term $I I I$, notice that $w \in A_{p / s^{\prime}} \subset A_{\infty}$; we thus get by Lemma 25 that

$$
I I I \leq\|A\|_{*} w(Q)^{1 / p} .
$$

Next we estimate $I V$. By Lemmas 21(c) and 25, we have

$$
\begin{aligned}
& \left|m_{\mathrm{Q}, w}(A)-m_{2^{j+1} \mathrm{Q}, w^{-s^{\prime} /\left(p-s^{\prime}\right)}}(A)\right| \\
& \quad \leq\left|m_{\mathrm{Q}, w}(A)-m_{\mathrm{Q}}(A)\right|+\left|m_{\mathrm{Q}}(A)-m_{2^{j+1} \mathrm{Q}}(A)\right| \\
& \quad+\mid m_{2^{j+1} \mathrm{Q}}(A)-m_{2^{j+1} \mathrm{Q}, w^{-s^{\prime} /\left(p-s^{\prime}\right)}(A) \mid \leq \frac{1}{w(Q)}} \\
& \quad \cdot \int_{Q}\left|A(t)-m_{\mathrm{Q}}(A)\right| w(t) d t+2^{n}(j+1)\|A\|_{*} \\
& \quad+\frac{1}{w^{-s^{\prime} /\left(p-s^{\prime}\right)}\left(2^{j+1} Q\right)} \\
& \quad \cdot \int_{2^{j+1} \mathrm{Q}}\left|A(t)-m_{2^{j+1} \mathrm{Q}}(A)\right| w^{-s^{\prime} /\left(p-s^{\prime}\right)}(t) d t \\
& \leq C(j+1)\|A\|_{*} \cdot
\end{aligned}
$$

Hence,

$$
I V \leq C(j+1)\|A\|_{*} w(Q)^{1 / p} .
$$

As a result,

$$
\begin{aligned}
& \frac{1}{w(Q)^{\kappa / p}}\left(\int_{Q} I I_{1}(y)^{p} w(y) d y\right)^{1 / p} \\
& \leq C\|A\|_{*}\|f\|_{L^{p, \kappa}(w)} \sum_{j=1}^{\infty}(j+1) \frac{w(Q)^{(1-\kappa) / p}}{w\left(2^{j+1} Q\right)^{(1-\kappa) / p}} \\
& \leq C\|A\|_{*}\|f\|_{L^{p, \kappa}(w)} .
\end{aligned}
$$

For $I_{2}$, by Hölder's inequality and $w \in A_{p / s^{\prime}}$, we get

$$
\begin{aligned}
& I I_{2} \leq C\|\Omega\|_{L^{s}} \\
& \cdot \sum_{j=1}^{\infty} \frac{\left|2^{j+2} Q\right|^{1 / s}}{\left|2^{j} Q\right|}\left(\int_{2^{j+1} Q}|f(z)|^{p} w(z) d z\right)^{1 / p} \\
& \cdot\left(\int_{2^{j+1} Q}\left|m_{2^{j+1} Q, w^{-s^{\prime} /\left(p-s^{\prime}\right)}}(A)-A(z)\right|^{p s^{\prime} /\left(p-s^{\prime}\right)}\right. \\
& \left.\cdot w(z)^{-s^{\prime} /\left(p-s^{\prime}\right)} d z\right)^{\left(p-s^{\prime}\right) / p s^{\prime}} \leq C\|\Omega\|_{L^{s}}\|A\|_{*} \\
& \cdot\|f\|_{L^{p, \kappa}(w)} \sum_{j=1}^{\infty} \frac{\left|2^{j+2} Q\right|^{1 / s}}{\left|2^{j} Q\right|} w\left(2^{j+1} Q\right)^{\kappa / p} \\
& \cdot w^{-s^{\prime} /\left(p-s^{\prime}\right)}\left(2^{j+1} Q\right)^{\left(p-s^{\prime}\right) / p s^{\prime}} \leq C\|A\|_{*}\|f\|_{L^{p, \kappa}(w)} \\
& \quad \sum_{j=1}^{\infty} \frac{1}{w\left(2^{j+1} Q\right)^{(1-\kappa) / p}} \cdot
\end{aligned}
$$

Therefore,

$$
\begin{aligned}
& \frac{1}{w(Q)^{\kappa / p}}\left(\int_{Q} I I_{2}^{p} w(y) d y\right)^{1 / p} \\
& \leq C\|A\|_{*}\|f\|_{L^{p, \kappa}(w)} \sum_{j=1}^{\infty} \frac{w(Q)^{(1-\kappa) / p}}{w\left(2^{j+1} Q\right)^{(1-\kappa) / p}} \\
& \quad \leq C\|A\|_{*}\|f\|_{L^{p, \kappa}(w)} .
\end{aligned}
$$

So far, we have completed the proof of (25).

Inequality (26) can be immediately obtained from (65) and (25).

Proof of Theorem 12. As before, we prove (27) at first. Assume $Q$ to be the same as in the proof of (22), denote $\bar{Q}=2 Q$, and set

$$
A_{\overline{\mathrm{Q}}}(y)=A(y)-m_{\overline{\mathrm{Q}}}(\nabla A) y .
$$

We also decompose $f$ according to $\overline{\mathrm{Q}}: f=f \chi_{\overline{\mathrm{Q}}}+f \chi_{(\overline{\mathrm{Q}})^{c}}:=$ $f_{1}+f_{2}$. Then we get

$$
\begin{aligned}
& \frac{1}{w(Q)^{\kappa / p}}\left(\int_{Q}\left|\widetilde{T}_{\Omega}^{A} f(y)\right|^{p} w(y) d y\right)^{1 / p} \\
& \leq \frac{1}{w(Q)^{\kappa / p}}\left(\int_{Q}\left|\widetilde{T}_{\Omega}^{A} f_{1}(y)\right|^{p} w(y) d y\right)^{1 / p} \\
& \quad+\frac{1}{w(Q)^{\kappa / p}}\left(\int_{Q}\left|\widetilde{T}_{\Omega}^{A} f_{2}(y)\right|^{p} w(y) d y\right)^{1 / p} \\
& :=I+I I .
\end{aligned}
$$


For I, Theorem 23 and Lemma 24(1) imply

$$
\begin{aligned}
I & \leq \frac{1}{w(Q)^{\kappa / p}}\left\|\widetilde{T}_{\Omega}^{A} f_{1}\right\|_{L^{p}(w)} \\
& \leq \frac{C}{w(Q)^{\kappa / p}}\|\Omega\|_{\infty}\|\nabla A\|_{*}\left\|f_{1}\right\|_{L^{p}(w)} \\
& \leq C\|\nabla A\|_{*}\|f\|_{L^{p, \kappa}(w)} \frac{w(\bar{Q})^{\kappa / p}}{w(Q)^{\kappa / p}} \\
& \leq C\|\nabla A\|_{*}\|f\|_{L^{p, \kappa}(w)} \cdot
\end{aligned}
$$

We will omit the proof for II since it is similar to and even easier than the part of $I I$ in the proof of (22), except that we use the conditions $w \in A_{p}, \Omega \in L^{\infty}\left(S^{n-1}\right), m=2$, and $f \in$ $L^{p, \kappa}(w)$. For inequality (28), it can be easily proved by (27) and (65). Thus, we complete the proof of Theorem 12.

\section{Competing Interests}

The authors declare that they have no competing interests.

\section{Acknowledgments}

The research was partially supported by the National Nature Science Foundation of China under Grant no. 11171306 and no. 11571306 and sponsored by the Scientific Project of Zhejiang Provincial Science Technology Department under Grant no. 2011C33012 and the Scientific Research Fund of Zhejiang Provincial Education Department under Grant no. Z201017584.

\section{References}

[1] J. Cohen and J. Gosselin, "A BMO estimate for multilinear singular integrals," Illinois Journal of Mathematics, vol. 30, no. 3, pp. 445-464, 1986.

[2] S. Hofmann, "On certain nonstandard Calderón-Zygmund operators," Studia Mathematica, vol. 109, no. 2, pp. 105-131, 1994.

[3] S. Z. Lu, H. X. Wu, and P. Zhang, "Multilinear singular integrals with rough kernel," Acta Mathematica Sinica (English Series), vol. 19, no. 1, pp. 51-62, 2003.

[4] Y. Ding and S. Z. Lu, "Weighted boundedness for a class of rough multilinear operators," Acta Mathematica Sinica-English Series, vol. 17, no. 3, pp. 517-526, 2001.

[5] S. Z. Lu and P. Zhang, "Lipschitz estimates for generalized commutators of fractional integrals with rough kernel," Mathematische Nachrichten, vol. 252, no. 1, pp. 70-85, 2003.

[6] C. B. Morrey, "On the solutions of quasi-linear elliptic partial differential equations," Transactions of the American Mathematical Society, vol. 43, no. 1, pp. 126-166, 1938.

[7] F. Chiarenza and M. Frasca, "Morrey spaces and HardyLittlewood maximal function," Rendiconti di Matematica e delle sue Applicazioni, vol. 7, no. 3-4, pp. 273-279, 1987.

[8] D. Fan, S. Lu, and D. Yang, "Regularity in Morrey spaces of strong solutions to nondivergence elliptic equations with VMO coefficients," Georgian Mathematical Journal, vol. 5, no. 5, pp. 425-440, 1998.
[9] J. Peetre, "On the theory of $L_{\rho, \lambda}$ spaces," Journal of Functional Analysis, vol. 4, no. 1, pp. 71-87, 1969.

[10] Y. Komori and S. Shirai, "Weighted Morrey spaces and a singular integral operator," Mathematische Nachrichten, vol. 282, no. 2, pp. 219-231, 2009.

[11] H. Wang, "The boundedness of some operators with rough kernel on the weighted Morrey spaces," Acta Mathematica Sinica, vol. 55, no. 4, pp. 589-600, 2012 (Chinese).

[12] M. Paluszynski, "Characterization of the Besov spaces via the commutator operator of Coifman, Rochberg and Weiss," Indiana University Mathematics Journal, vol. 44, no. 1, pp. 1-17, 1995.

[13] R. A. DeVore and R. C. Sharpley, "Maximal functions measuring smoothness," Memoirs of the American Mathematical Society, vol. 47 , no. 293, 1984.

[14] Y. Ding and S. Z. Lu, "Weighted norm inequalities for fractional integral operators with rough kernel," Canadian Journal of Mathematics, vol. 50, no. 1, pp. 29-39, 1998.

[15] L. Grafakos, Modern Fourier Analysis, vol. 250 of Graduate Texts in Mathematics, Springer, New York, NY, USA, 2nd edition, 2009.

[16] S. Z. Lu, Y. Ding, and D. Y. Yan, Singular Integrals aand Related Topics, World Scientific, 2007.

[17] B. Muckenhoupt and R. L. Wheeden, "Weighted bounded mean oscillation and the Hilbert transform," Studia Mathematica, vol. 54, no. 3, pp. 221-237, 1976. 


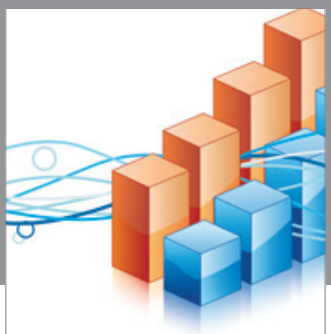

Advances in

Operations Research

vatem alat4

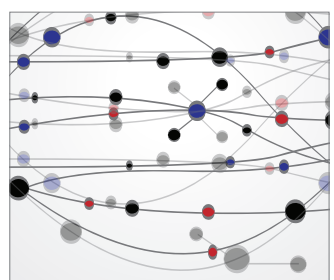

\section{The Scientific} World Journal
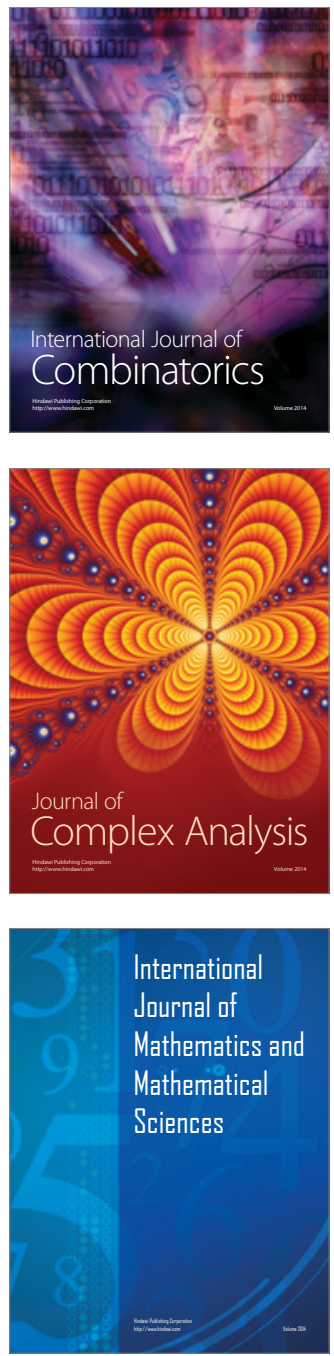
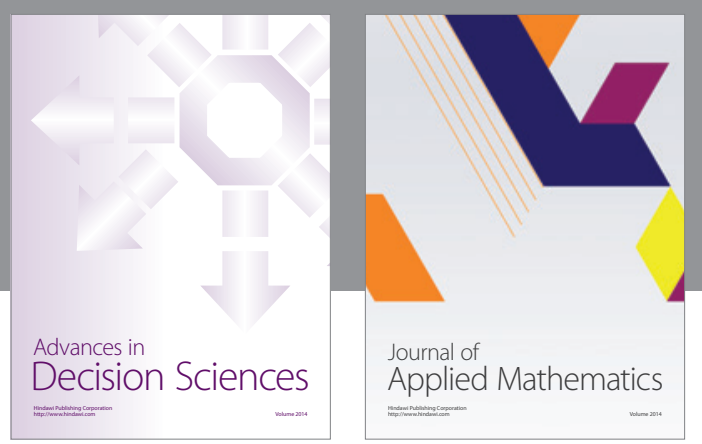

Algebra

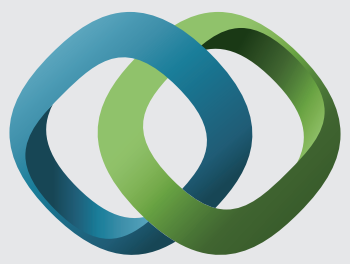

\section{Hindawi}

Submit your manuscripts at

http://www.hindawi.com
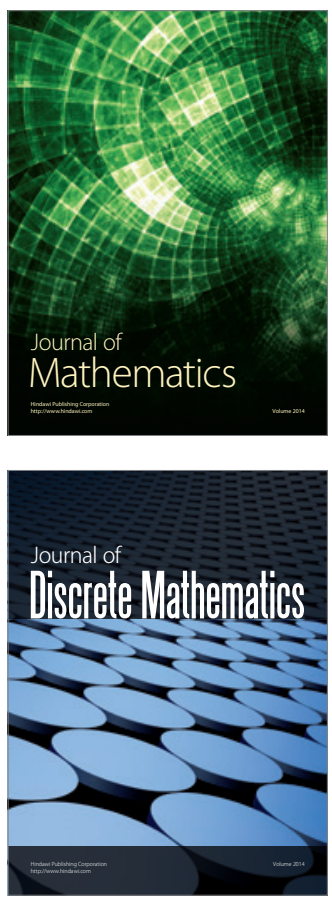

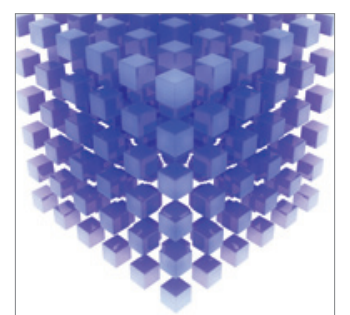

Mathematical Problems in Engineering
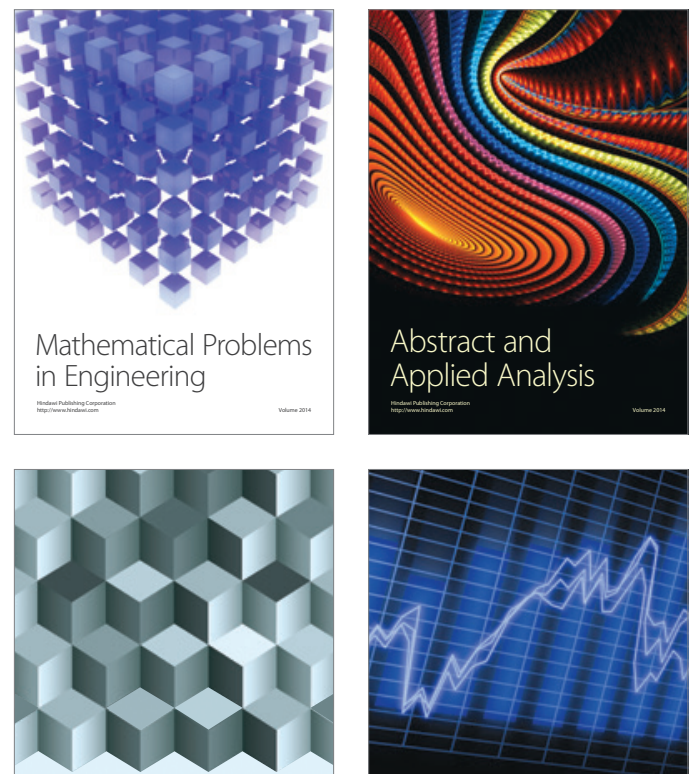

Journal of

Function Spaces

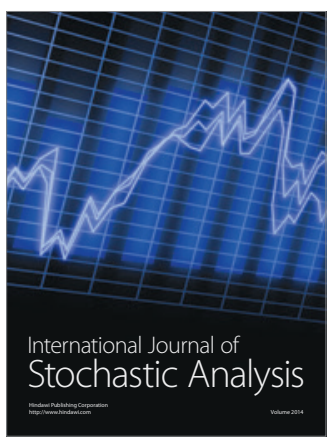

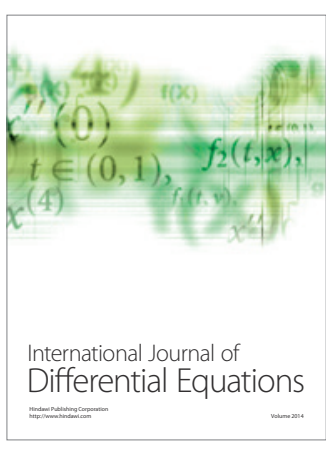
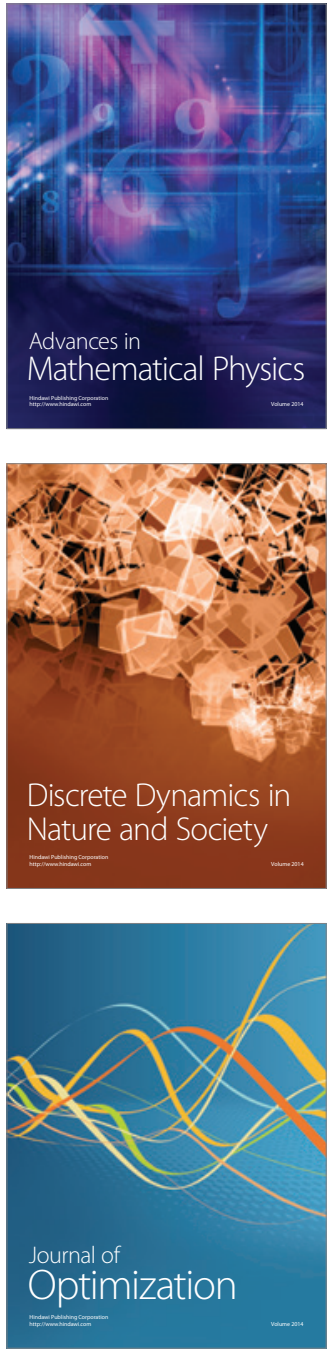